\title{
AVALIÇÃO DO PONTO DE NÉVOA E DO PONTO DE FLUIDEZ DO BIODIESEL DE OURICURI E DAS SUAS MISTURAS COM DIESEL
}

\author{
R. GABRIEL ${ }^{1}$, A. K. S. LIMA ${ }^{1}$, J. I. SOLETTI ${ }^{1}$,S . H. V. de CARVALHO ${ }^{1}$ e A. S. PEITER ${ }^{2}$ \\ ${ }^{1}$ Universidade Federal de Alagoas, Centro de Tecnologia \\ ${ }^{2}$ Universidade Federal de Alagoas, Campus do Sertão, Unidade Santana do Ipanema \\ E-mail para contato: jisoletti@gmail.com
}

\begin{abstract}
RESUMO - Atualmente o biodiesel é comercializado junto ao diesel constituindo as misturas (blendas) de biodiesel em conformidade com o Programa Nacional de Produção e Uso de Biodiesel (Lei $n^{\circ}$ 11.097/05). A depender do tipo de biodiesel utilizado, haverá alteração das propriedades físico-química das misturas. Dentre as propriedades, tem-se a cristalização que pode inviabilizar sua utilização em temperaturas frias, devido a deposição de ésteres graxos e parafinas no motor, provocando a queda das propriedades de fluxo. Neste trabalho foi analisado o ponto de fluidez e o ponto de névoa em diferentes proporções das misturas de diesel - biodiesel de ouricuri (B0, B20, B40, B60, B80 e B100), para diferentes temperaturas, de acordo com a norma brasileira NBR 11349:1990. Como resultado, obteve-se a não adequação do uso do biodiesel de ouricuri a temperaturas inferiores à $21^{\circ} \mathrm{C}$, principalmente nas misturas B60, B80 e B100 as quais contém maior concentração de biodiesel, observando-se a formação de cristais.
\end{abstract}

\section{INTRODUÇÃO}

Para contornar o problemas de emissões de gases de efeito estufa e da poluição gerada pela utilização de combustíveis derivados do petróleo, vários países implementaram a utilização de misturas de diesel / biodiesel. No Brasil, através da resolução $\mathrm{n}^{\circ}$ 7/2008 a ANP estabeleceu que desde Janeiro de 2010, é obrigatória a adição de biodiesel ao óleo diesel comercializado no país na proporção de $5 \%$ em volume. Em comparação com o diesel, o biodiesel produz menos emissões de poluentes nocivos. Além disso, tem lubrificação melhorada, menor toxicidade, maior ponto de fulgor e biodegradabilidade melhorada segundo os autores Nazario (2014), Souza et al.(2013) e Pidol et al. (2012).

Existe, atualmente, uma série de pesquisas e testes voltados à utilização de biodiesel no Brasil e no mundo, Caíres et al. (2014), Chhetti et al. (2012), Meng et al. (2014), Zarska et al. (2014).

Segundo Ferella et al. (2010), a conversão de óleo bruto para a forma de éster é normalmente realizada para reduzir a viscosidade do óleo e eliminar problemas que surgem durante seu uso em motores a diesel. A transesterificação de óleos vegetais assume importância estratégica para o setor 


\section{9 a 22 de outubro de 2014 \\ Florianópolis/SC}

energético, uma vez que os ésteres produzidos a partir de óleos vegetais e alcoóis de cadeia curta (ou seja, o biodiesel) modificam as propriedades originais do óleo adequando-o ao uso em motores diesel e tornando-os potenciais substitutos do óleo diesel mineral. Muitos óleos vegetais podem ser usados para a produção de biodiesel e a escolha da matéria- prima deve ser com base em considerações técnicas e econômicas.

O uso das misturas diesel/biodiesel pode afetar diversas propriedades físico-químicas do combustível, tais como: viscosidade, ponto de fluidez, ponto de nevoa, densidade, número de cetano, cor, calor de combustão, teor de enxofre, volatilidade, estabilidade à oxidação, lubricidade e turbidez conforme, Kozak et al (2013). Para garantir a padronização e identidade do biodiesel é necessário estabelecer padrões de qualidade, que permitam o monitoramento e evitem prejudicar a qualidade das emissões da queima, desempenho e a integridade do motor, assim como a segurança no transporte e manuseio. No Brasil, a ANP é responsável pela definição de padrões para a certificação da qualidade do biodiesel, Souza et al. (2013), Fernandes et al. (2011).

Segundo Hoekman et al. (2012), em baixas temperaturas, o biodiesel e suas misturas com óleo diesel derivado de petróleo, apresentam a deposição de ésteres graxos e parafinas durante o armazenamento, transporte e sua utilização, ocasionando queda das propriedades de fluxo, um dos problemas graves e conhecidos de longa data na indústria do petróleo. A baixas temperaturas ocorre a formação de núcleos de cristais parafínicos, visíveis a olho nu. A temperatura em que os cristais se tornam visíveis é definida como ponto de névoa. Nas temperaturas abaixo do ponto de névoa, o crescimento cristalino e a aglomeração continuam até que os cristais fiquem grandes o bastante para restringir ou impedir o fluxo livre dos combustíveis nas tubulações e nos filtros. Essa propriedade é conhecida como ponto de fluidez do biodiesel que é definido como a mais baixa temperatura em que o líquido escoa livremente. A tendência à solidificação está relacionada com a matéria-prima que deu origem ao biodiesel, e à sua composição.

O ponto de fluidez e o ponto de névoa são usados para caracterizar a operacionalidade do escoamento de combustível em baixas temperaturas, porque afeta o emprego do combustível, especialmente em condições de clima frio. O ponto de fluidez do biodiesel é mais elevado devido maior quantidade de ácidos graxos saturados. A forma alternativa para superar os problemas de escoamento do biodiesel em baixas temperaturas é misturá-lo com óleo diesel. Logo, o funcionamento em baixas temperaturas deve ser levado em consideração uma vez que, assim como para o diesel convencional, devem ser tomadas precauções para garantir a operacionalidade à baixas temperaturas de biodiesel e suas misturas.

Neste trabalho são apresentados dados necessários ao monitoramento do ponto de fluidez e do ponto de névoa das misturas diesel/biodiesel: B0, B20, B40, B60, B80 e B100 para o biodiesel produzido a partir do óleo de ouricuri.

\section{METODOLOGIA}

Para a produção do biodiesel de Ouricuri os reagentes utilizados foram álcool etílico absoluto P.A., hidróxido de sódio P.A. e óleo da semente de Ouricuri. A reação foi realizada sob as seguintes 
condições: razão molar óleo/álcool de 1:10, quantidade de catalisador de $1 \%$ referente a massa de óleo, tempo de reação de 60 minutos e temperatura de $70{ }^{\circ} \mathrm{C}$. A reação de transesterificação ocorreu em uma unidade piloto composta por um reator, um agitador mecânico e por um banho termostático (Modelo TE - 184, TECNAL) no Laboratório de Sistemas de Separação e Otimização de Processos (LASSOP) situada na Universidade Federal de Alagoas, conforme Figura 1.

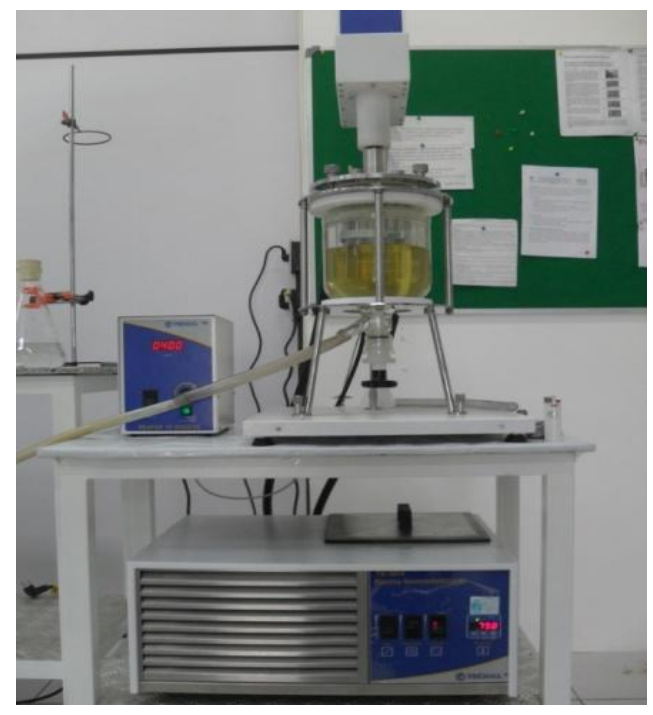

Figura 1- Unidade piloto utilizada.

Após a produção e purificação do biodiesel, foram realizadas as misturas (blendas) diesel/biodiesel, para as concentrações pré-estabelecidas: B20, B40, B60, B80, B100, para finalmente realizar a medida de ponto de fluidez e o ponto de névoa.

O método para determinação do ponto de fluidez consiste em resfriar uma amostra num ritmo pré-determinado, observando-se a sua fluidez a cada queda de temperatura de $3^{\circ} \mathrm{C}$, até que visualmente a superfície da amostra permaneça imóvel (ponto de congelamento) por 5 segundos, ao se colocar o tubo de ensaio com a amostra, na posição horizontal. A temperatura $3^{\circ} \mathrm{C}$ acima desta é o ponto de mínima fluidez. A verificação do ponto de fluidez será feita de acordo com o emprego da norma brasileira NBR 11349:1990.

O método para determinação do ponto de nevoa foi obtido pela norma ASTM D2500, onde observou-se a temperatura do combustível, em um processo de resfriamento, onde se observou formação dos primeiros cristais. 


\section{9 a 22 de outubro de 2014 \\ Florianópolis/SC}

\section{RESULTADOS E DISCUSSÕES}

O processo de mistura para a produção de blendas, é possível e recomendado, a fim de melhorar a qualidade do combustível e por isso tem sido amplamente estudada. No entanto, a mistura biodiesel/diesel é uma mistura muito complexa. Estão presentes no biodiesel compostos de ésteres, glicerídeos, pigmentos e antioxidantes. Os compostos no diesel são naftênicos, parafínicos e aromáticos, em diferentes proporções. Devido à mistura, várias alterações podem ser percebidas, dentre estas o ponto de fluidez e o ponto de névoa. A verificação do ponto de fluidez foi feita de acordo com o emprego da norma brasileira NBR 11349:1990 e o para o ponto de névoa utilizou-se a norma ASTM D2500.

Segundo Lobo et al. (2009), na Europa, os valores para o ponto de fluidez e para o ponto de névoa, devem ser estabelecidos por cada país em função do seu clima. Nos Estados Unidos, o valor do ponto de fluidez é dependente da sazonalidade do clima. No Brasil, é estabelecida a determinação do ponto de entupimento de filtro a frio para o biodiesel, conforme os métodos ABNT NBR 14747, EN ISO 116 e ASTM D6371. O valor máximo de $19^{\circ} \mathrm{C}$ estabelecido na Resolução brasileira é aplicável para as regiões Sul, Sudeste, Centro-Oeste e para o estado da Bahia, devendo ser anotado para as demais regiões.

Estas informações são de grande importância para avaliar a aplicabilidade do combustível em regiões de clima frio. Quanto maior for o tamanho da cadeia e/ou o caráter saturado das moléculas do biodiesel, mais alto serão os valores destes parâmetros. É de se esperar, portanto, que o biodiesel originário de gordura animal apresente valores mais elevados que o biodiesel proveniente de gordura vegetal, devido ao seu alto teor de ácidos graxos saturados.

De uma forma geral o biodiesel a baixas temperaturas, tende a solidificar-se parcialmente ou a perder sua fluidez, levando à interrupção do fluxo, ocasionando problemas na partida do motor. A Tabela 1 indica o comportamento físico do biodiesel de ouricuri com o aumento da temperatura. Destes ensaios, pode-se analisar o ponto de fluidez, assim como o ponto de névoa, que é a temperatura de um combustível em um processo de resfriamento, onde se observa a formação dos primeiros cristais.

Conforme a Tabela 1, vemos que os blends de biodiesel/diesel e o biodiesel de ouricuri congelaram à $0^{\circ} \mathrm{C}$. Com isso pra as misturas B20, B40, B60, B80 e B100 obtemos o ponto de fluidez igual a $3^{\circ} \mathrm{C}$, pois o ponto de fluidez corresponde a temperatura do ponto de congelamento mais $3^{\circ} \mathrm{C}$. No entanto à $3^{\circ} \mathrm{C}$, as misturas B20, B40, B60, B80 e B100 não se mantiveram estáticas, por um período de 5 segundos, ao colocar o tubo de ensaio contendo a amostra na posição horizontal.

Seguimos observando-se o comportamento das misturas, aumentando-se gradativamente a temperatura até $24^{\circ} \mathrm{C}$, com uma variação de $3^{\circ} \mathrm{C}$ à cada nova temperatura. Neste ponto, foi possível perceber que todas as misturas estavam totalmente no seu estado liquido. 
Tabela 1 - Congelamento da mistura de diesel /biodiesel etílico de ouricuri.

\begin{tabular}{|c|c|c|c|c|c|c|}
\hline \multicolumn{7}{|c|}{ BLENDS DE BIODIESEL/DIESEL DE OURICURI } \\
\hline \multicolumn{3}{|c|}{ Temperatura $0^{\circ} \mathrm{C}$} & \multicolumn{2}{|c|}{ Temperatura $3^{\circ} \mathrm{C}$} & \multicolumn{2}{|c|}{ Temperatura $6^{\circ} \mathrm{C}$} \\
\hline Blends & Congelamento & Observação & Congelamento & Observação & Congelamento & Observação \\
\hline B0 & $\mathrm{NC}$ & TL & $\mathrm{NC}$ & TL & $\mathrm{NC}$ & TL \\
\hline B20 & $\mathrm{C}$ & $\mathrm{AM}$ & $\mathrm{NC}$ & TL & $\mathrm{NC}$ & FC \\
\hline B40 & $\mathrm{C}$ & $\mathrm{AM}$ & $\mathrm{C}$ & $\mathrm{E}$ & $\mathrm{NC}$ & FC \\
\hline B60 & $\mathrm{C}$ & $\mathrm{AM}$ & $\mathrm{C}$ & $\mathrm{E}$ & $\mathrm{C}$ & $\mathrm{E}$ \\
\hline B80 & $\mathrm{C}$ & AM & $\mathrm{C}$ & $\mathrm{E}$ & $\mathrm{C}$ & $\mathrm{E}$ \\
\hline B100 & $\mathrm{C}$ & $\mathrm{AM}$ & $\mathrm{C}$ & $\mathrm{E}$ & $\mathrm{C}$ & $\mathrm{E}$ \\
\hline \multicolumn{3}{|c|}{ Temperatura $9^{\circ} \mathrm{C}$} & \multicolumn{2}{|c|}{ Temperatura $12^{\circ} \mathrm{C}$} & \multicolumn{2}{|c|}{ Temperatura $15^{\circ} \mathrm{C}$} \\
\hline Blends & Congelamento & Observação & Congelamento & Observação & Congelamento & Observação \\
\hline B0 & $\mathrm{NC}$ & $\mathrm{TL}$ & $\mathrm{NC}$ & $\mathrm{TL}$ & $\mathrm{NC}$ & $\mathrm{TL}$ \\
\hline B20 & $\mathrm{NC}$ & $\mathrm{TL}$ & $\mathrm{NC}$ & TL & $\mathrm{NC}$ & $\mathrm{TL}$ \\
\hline B40 & $\mathrm{NC}$ & FC & $\mathrm{NC}$ & FC & $\mathrm{NC}$ & TL \\
\hline B60 & $\mathrm{NC}$ & FC & $\mathrm{NC}$ & FC & $\mathrm{NC}$ & FC \\
\hline B80 & $\mathrm{C}$ & $\mathrm{E}$ & $\mathrm{C}$ & $\mathrm{E}$ & $\mathrm{NC}$ & FC \\
\hline B100 & $\mathrm{C}$ & $\mathrm{E}$ & $\mathrm{C}$ & $\mathrm{E}$ & $\mathrm{NC}$ & FC \\
\hline \multicolumn{3}{|c|}{ Temperatura $18^{\circ} \mathrm{C}$} & \multicolumn{2}{|c|}{ Temperatura $21^{\circ} \mathrm{C}$} & \multicolumn{2}{|c|}{ Temperatura $24^{\circ} \mathrm{C}$} \\
\hline Blends & Congelamento & Observação & Congelamento & Observação & Congelamento & Observação \\
\hline B0 & $\mathrm{NC}$ & TL & $\mathrm{NC}$ & TL & $\mathrm{NC}$ & $\mathrm{TL}$ \\
\hline B20 & $\mathrm{NC}$ & TL & $\mathrm{NC}$ & TL & $\mathrm{NC}$ & TL \\
\hline B40 & $\mathrm{NC}$ & TL & $\mathrm{NC}$ & TL & $\mathrm{NC}$ & TL \\
\hline B60 & $\mathrm{NC}$ & FC & $\mathrm{NC}$ & FC & $\mathrm{NC}$ & TL \\
\hline B80 & $\mathrm{NC}$ & FC & $\mathrm{NC}$ & FC & $\mathrm{NC}$ & TL \\
\hline B100 & $\mathrm{NC}$ & FC & $\mathrm{NC}$ & FC & $\mathrm{NC}$ & TL \\
\hline
\end{tabular}

${ }^{*} \mathrm{C}=$ congelou, $\mathrm{NC}=$ não congelou, $\mathrm{AM}=$ ausência de movimento, $\mathrm{E}=$ escorreu, $\mathrm{FC}=$ formação de cristais e $\mathrm{TL}=$ totalmente líquido.

Com a redução da temperatura ocorre a formação de núcleos de cristais parafínicos invisíveis a olho nu. O crescimento desses cristais aumenta com a redução da temperatura. Os cristais tornam-se visíveis na temperatura definida como o ponto de névoa, porque os cristais formam suspensão enevoada. Conforme dados experimentais da Tabela 1, o ponto de nevoa da mistura B20 foi alcançado na temperatura de $6{ }^{\circ} \mathrm{C}$, o da mistura $\mathrm{B} 40$ foi à $12{ }^{\circ} \mathrm{C}$ e das misturas B60, B80 E B100 foi de $21^{\circ} \mathrm{C}$ onde observou-se turbidez/formação dos primeiros cristais no tubo de ensaio.

Em temperaturas abaixo do ponto de névoa, cristais maiores fundem-se uns aos outros e formam grandes aglomerados que podem restringir ou impedir o escoamento do combustível em tubulações e filtros. Esse comportamento pode dificultar a partida do motor ou o seu desempenho em horários mais frios. Portanto, essas propriedades desfavorecem o uso de óleos vegetais in natura em motores do ciclo diesel, particularmente em regiões de clima temperado, pois os óleos vegetais 


\section{9 a 22 de outubro de 2014 \\ Florianópolis/SC}

normalmente apresentam ponto de fluidez superior ao do diesel mineral convencional. Alternativamente, podem ser utilizados inibidores de cristalização, de forma a permitir o uso e estocagem destes combustíveis em regiões mais frias e a utilização de aditivos apropriados no óleo vegetal pode conferir-lhe maior fluidez, diminuindo seu ponto de fluidez. Vale ressaltar que este problema não é exclusivo do biodiesel, pois o diesel de petróleo contém parafinas que apresentam tipicamente o mesmo comportamento.

\section{CONCLUSÃO}

Este estudo é de extrema importância para avaliar a aplicabilidade do biodiesel de ouricuri, assim como de suas blendas em regiões de clima frio. Quanto maior a porcentagem de biodiesel na mistura, mais a mesma sofre influências negativas com temperaturas baixas, fazendo-se necessária a adição de anti-congelantes e outros aditivos para sua utilização em climas frios. No caso de não haver a adição de anti-congelantes, e seguindo as especificações da ANP, poderíamos utilizar o biobiesel de Ouricuri apenas para misturas abaixo de B60 onde obtivemos uma temperatura de $21^{\circ} \mathrm{C}$.

\section{REFERÊNCIAS}

CAIRES, A.R.L.; SCHERER, M.D.; SOUZA, J.E.; OLIVEIRA, S.L.; M'PEKO, J.C. The role of viscosity in the fluorescence behavior of the diesel/biodiesel blends. Renewable Energy 63, p. 388e391, 2014.

CHHETRI ,A.B.; WATTS, K.C. Viscosities of canola, jatropha and soapnut biodiesel at elevated temperatures and pressures. Fuel 102, p. 789-794, 2012.

FERELLA, F.; CELSO, G. M.; MICHELIS, I.; STANISCI, V.; VEGLIO, F. Optimization of the transesterification reaction in biodiesel production. Fuel, v. 89, p. 36-42, 2010.

FERNANDES, D.D.S.; GOMES, A.A.; COSTA, G.B.; SILVA, G.W.B.; VERAS, G. Determinations of biodiesel contente in biodiesel/diesel blends using NIR and visible spectroscopy with variable selection. Elsevier Talanta 87 (2011) 30-34.

HOEKMAN, S.K.; BROCH, A.; ROBBINS, C.; CENICEROS, E.; NATARAJAN, M. Review of biodiesel composition, properties, and specifications. Elsevier Renenwable and Sustainable Energy Reviews 16, p. 143-169, 2012.

LOBO, I.P.; FERREIRA, S.L.C.; CRUZ, R.S. Biodiesel: parâmetros de qualidade e métodos analíticos. Quim. Nova, Vol. 32, No. 6, 1596-1608, 2009.

KOZAK, L.; NIEDZIELKI, P.; WACHOWIAK, W. The tandem analytical method of flow injection diode array spectrophotometry end flame atomic absorption spectrometry (FI-DAD(vic)FAAS) in iron speciation studies using 1, 10-ohenanthroline complexes. Microchemical Journal, 2013. 
MENG, X.; JIA.M.; WANG, T. Neural network prediction of biodiesel kinematic viscosity at 313 K. Fuel 121, p. 133-140, 2014.

NAZARIO, J. L. Avaliação do Efeito da Agitação e Mistura no Processo de Produção do Biodiesel de Mamona via Transesterificação Metílica. Dissertação de mestrado. Universidade Federal de Alagoas - UFAL. Maceió - AL, 2014.

PIDOL, L.; LECOINTE, B.; STARCK, L.; JEULAND, N. Ethanol-biodiesel-Diesel fuel blends: Performances and emissions in conventional Diesel and advanced Low Temperature combustions. Elsevier Fuel 93 (2012) 329-338.

SOUZA, J.E.; SCHERER, M. D.; CACERES, J.A.S.; CAIRES, A.R.L.; M'PEKO, J.C. A close dielectric spectroscopia analysis of diesel/biodiesel blends and potential dielectric approaches for biodiesel content assessment. Elsevier Fuel 105, p. 705-710, 2013.

ZARSKA, M.; BARTOSZEK, K.; DZIDA, M. High pressure physicochemical properties of biodiesel components derived from coconut oil or babassu oil. Fuel 125, p. 144-151, 2014. 